\title{
5-French SOFIA: Safe Access and Support in the Anterior Cerebral Artery, Posterior Cerebral Artery, and Insular Middle Cerebral Artery
}

\author{
Bradley A. Gross ${ }^{a, b} \quad$ William J. Ares ${ }^{a, b}$ Cynthia L. Kenmuira, c \\ Ashutosh P. Jadhav ${ }^{a, c}$ Tudor G. Jovin ${ }^{a, c}$ Brian T. Jankowitz ${ }^{a, b}$ \\ a Stroke Institute, University of Pittsburgh Medical Center, Pittsburgh, PA, USA; ${ }^{b}$ Department \\ of Neurological Surgery, University of Pittsburgh Medical Center, Pittsburgh, PA, USA; \\ 'Department of Neurology, University of Pittsburgh Medical Center, Pittsburgh, PA, USA
}

\section{Keywords}

Intermediate catheter · Stroke · Aneurysm · Flow diversion · Pipeline · Arteriovenous malformation - Embolization

\begin{abstract}
Introduction: Distal access catheters are an infrequent focus of technical notes in neurointervention. The 5-French SOFIA's unique design allows for compatibility with 6-French guide catheters, while its supple construction allows for remarkably distal access for a catheter with a 0.055 -inch inner diameter. Methods: The authors reviewed a prospectively maintained endovascular database for cases utilizing the 5-French SOFIA from February 2017 through November 2017. Case type, SOFIA location, microcatheter used, and catheter-related complications were noted. Results: Over the evaluated period, the 5-French SOFIA was utilized in 33 cases, including 13 aneurysm treatments, 10 arteriovenous shunt embolizations, 5 stroke thrombectomies, and 5 other cases. Of 5 flow diversion cases, 1 was for a symptomatic cavernous internal carotid artery aneurysm necessitating transradial access, another for a ruptured A3 aneurysm, and another for a middle cerebral artery (M2) aneurysm; 2 were more proximal aneurysms. Thrombectomies were for M2 $(n=3)$ or A2 $(n=2)$ occlusions. In all cases, the 5-French SOFIA reached its anticipated distal target without complication or the need to utilize a smaller/alternative catheter. Of these 33 cases, there were 10 cases of distal SOFIA target locations: $6 \mathrm{M} 2 / \mathrm{M} 3,3$ anterior cerebral arteries (ACA), and 1 posterior cerebral artery (PCA). M2/M3 and PCA catheterization was achieved over 2.1-Fr microcatheters; ACA
\end{abstract}


Gross et al:: 5F SOFIA

catheterization employed a 2.9-Fr microcatheter for pipeline embolization and a deployed stentriever in the setting of two thrombectomies. Conclusion: The 5-French SOFIA can be safely utilized for distal, superselective catheterization in the context of complex neurointervention, including aneurysm and arteriovenous shunt embolization and distal thrombectomy.

(c) 2018 S. Karger AG, Base

\section{Introduction}

While stroke thrombectomy has shifted the focus of the role of intermediate catheters to reperfusion [1], intermediate catheter design for complex neurointervention remains an important field for creative innovation. Many intermediate/reperfusion catheters are designed to be compatible with 6-French sheaths with outer diameters that may preclude catheterization of smaller distal vessels. The 5-French Soft torqueable catheter Optimized For Intracranial Access (5F SOFIA) is unique in its compatibility with 6-French guide catheters in contrast to many intermediate/reperfusion catheters with similar inner diameters. Though subjective, it is additionally known to be extremely soft and supple, with a recent paper demonstrating the ability to pass SOFIA into the intracranial circulation without even guidewire or microcatheter support: the "SNAKE" technique [2]. In this report, we review our recent experience with the $5 \mathrm{~F}$ SOFIA to illustrate how its construction allows for versatile usage in complex neurointerventional procedures.

\section{Methods}

The authors evaluated a prospectively maintained endovascular database for cases utilizing the $5 \mathrm{~F}$ SOFIA from February 2017 through November 2017. Prior to this period, we had only utilized the 6-French SOFIA for stroke thrombectomy. We noted case type, guide catheter/support for the SOFIA, final location of the SOFIA, indications for SOFIA usage, microcatheter utilized, and overall results/complications.

\section{Results}

Over the evaluated 9-month period, 33 patients were treated utilizing the 5F SOFIA catheter (Table 1). This included 8 aneurysm coilings/embolizations, 5 aneurysm flow diversion treatments, 10 arteriovenous shunt embolizations, 5 distal vessel occlusion thrombectomies, 2 tumor embolizations, 2 vasospasm treatments, and 1 epistaxis embolization. In all cases, the SOFIA reached its intended distal target, including 11 cases with the support of a 6-French, 0.071-inch guide catheter instead of a 6-French, long sheath. Anterior circulation target locations were: the intracranial internal carotid artery (ICA) $(n=8 ; 4$ supraclinoid ICA), the middle cerebral artery (M1) $(n=2), \mathrm{M} 2 / 3(n=6)$, and the anterior cerebral artery (ACA) $(n=3 ; 2$ A2). Posterior circulation target locations were: V3 $(n=6)$, V4 $(n=1)$, the basilar artery $(n=2)$, and the posterior cerebral artery (PCA) $(n=1)$. External carotid artery target locations were: the distal internal maxillary artery $(n=2)$ and the occipital artery $(n=2)$. In 18 cases, the SOFIA was specifically utilized in anticipation of a distal intermediate catheter target location, and in 6 cases, it was utilized due to visualized significant access vessel tortuosity. The SOFIA was most commonly advanced to its target over 2.1-Fr microcatheters (headway duo, $n=12$; echelon-10, $n=4$ ). In the remaining cases, larger microcatheters were utilized in the context of their need to treat the underlying pathology (2.4-Fr catheter for selected aneurysm coilings or 2.9/3.2-Fr microcatheters for aneurysm flow diversion or 
Table 1. 5F SOFIA final location stratified by pathology ${ }^{\mathrm{a}}$

\begin{tabular}{llll}
\hline & $n$ & SOFIA location & Microcatheter \\
\hline Aneurysm embolization & 8 & 5 supraclinoid ICA & 4 SL-10 \\
& & 1 cavernous ICA & 1 echelon-10 \\
& & $1 \mathrm{M} 2$ & 3 headway duo (Onyx) \\
& & $1 \mathrm{~V} 3$ & \\
\hline Aneurysm flow diversion & 5 & 2 cavernous ICA & 2 XT-27 \\
& & $1 \mathrm{~V} 3$ & 2 Marksman \\
& & $1 \mathrm{M} 1$ & 1 Phenom \\
\hline Arteriovenous malformation & 6 & $1 \mathrm{~A} 1-2$ junction & \\
embolization & & $2 \mathrm{M} 2 / 3$ & 5 headway duo \\
& & 2 basilar & 1 echelon-10 \\
\hline Arteriovenous fistula & & 1 P1 & \\
embolization & 4 & $3 \mathrm{~V} 3$ & 3 headway duo \\
\hline Stroke thrombectomy & 5 & $3 \mathrm{M} 2$ & 1 Scepter balloon \\
\hline
\end{tabular}

ICA, internal carotid artery.

a Excluding 5 cases: 2 tumor embolizations, 2 angioplasty for vasospasm, and 1 epistaxis embolization.

stroke thrombectomy). There were no cases of device failure in the 33 cases; it was never swapped for an alternative distal access catheter for improved support/better distal access. There were no dissections, perforations, or complications related to advancement of the SOFIA.

\section{Aneurysm Treatment}

The SOFIA catheter was utilized in 8 cases of aneurysm embolization. This reflects a small minority of aneurysms treated over the time period, as saccular aneurysms are most often treated with larger guide catheters to allow for balloon or stent-assisted coiling. In rare cases, where balloon-assisted coiling was not anticipated $(n=5)$, the SOFIA 0.055 was utilized and advanced over a 2.4- or 2.1-Fr coiling microcatheter. In 3 cases, the SOFIA was advanced over, and utilized to support, a 2.1-Fr headway duo microcatheter utilized for Onyx embolization of distal aneurysms. Two were mycotic aneurysms of distal middle cerebral artery (MCA) branches treated with the SOFIA tracked to, and maintained in, the M2.

The 5F SOFIA catheter was utilized in 5 cases of aneurysm flow diversion during the evaluated period; this included 2 ICA aneurysms, 1 V4 aneurysm, 1 M2 aneurysm, and 2 adjacent distal ACA aneurysms. Usage was always in the context of either a distal intermediate catheter target location or complex access anatomy, as we utilize larger inner diameter, stiffer intermediate catheters for more routine cases. In all cases, the SOFIA was advanced to its desired location over the 0.027 -inch microcatheter utilized to deploy the flow diverter. Illustrative cases include adjacent distal ACA fusiform aneurysms and a cavernous ICA aneurysm treated via transradial access with a 6-Fr guide catheter supporting the SOFIA (Fig. 1). 


\section{Interventional Neurology}

\begin{tabular}{l|l}
\hline Intervent Neurol 2018;7:308-314 \\
\hline DOI: 10.1159/000488253 & $\begin{array}{l}\text { (c) 2018 S. Karger AG, Basel } \\
\text { www.karger.com/ine }\end{array}$ \\
\hline
\end{tabular}

Gross et al.: 5F SOFIA

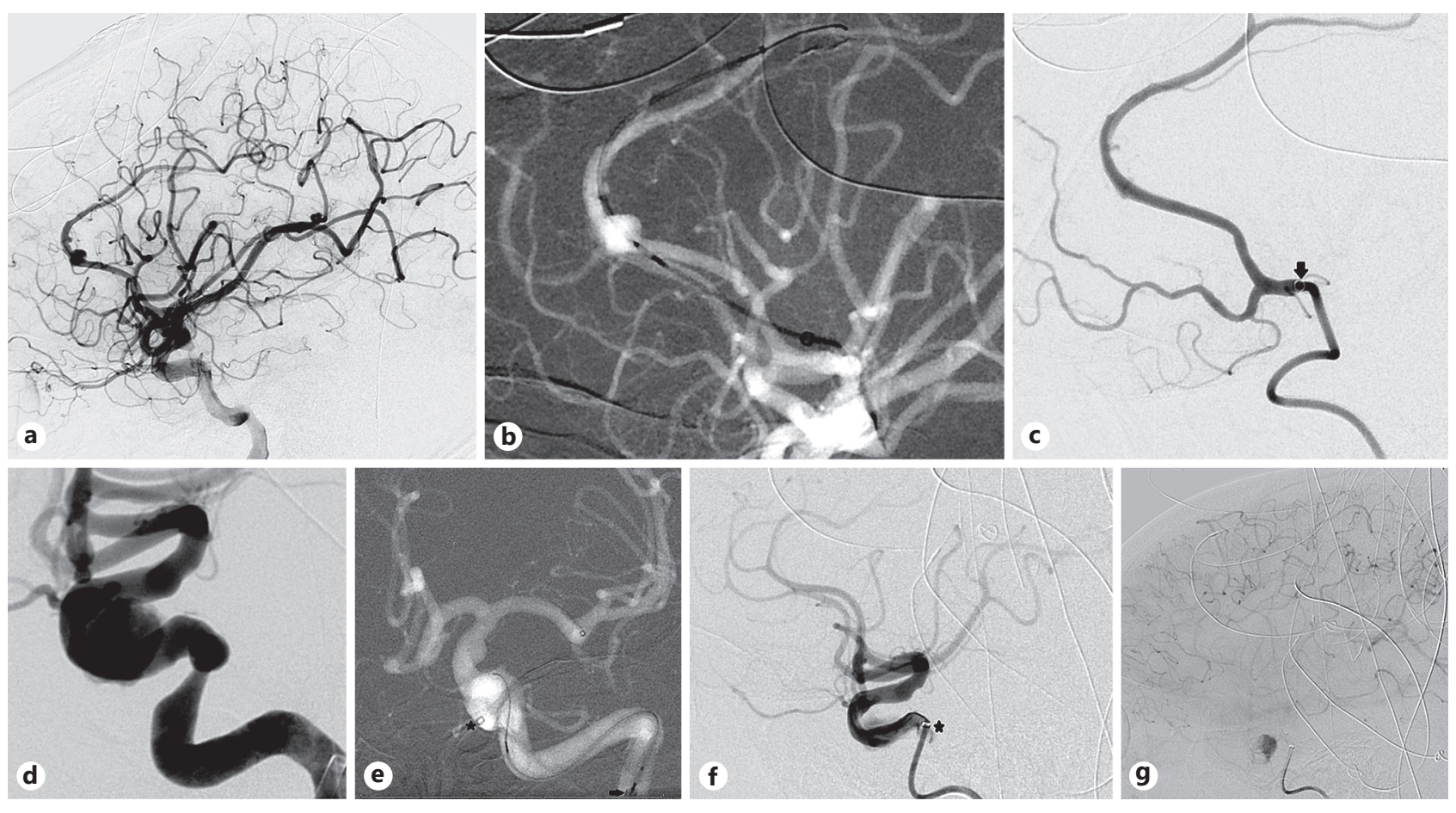

Fig. 1. Ruptured distal anterior cerebral artery (ACA) and adjacent distal ACA aneurysms (lateral angiographic view, a) treated via deployment of 2 telescoping pipeline embolization devices (lateral working view during pipeline advancement, b). A post-deployment superselective ACA run via the 5F SOFIA demonstrates immediate effect from the devices (c, note SOFIA location in ACA, arrow). This progressively symptomatic large cavernous aneurysm (lateral angiographic view, d) was treated via transradial access with a short 6-Fr sheath, a 6-Fr guide catheter (e, arrow), and 5F SOFIA (e, star) via pipeline flow diversion. A post-deployment lateral internal carotid artery run demonstrates the final location of the SOFIA catheter (f, star) and contrast stagnation in the aneurysm $(\mathbf{g})$.
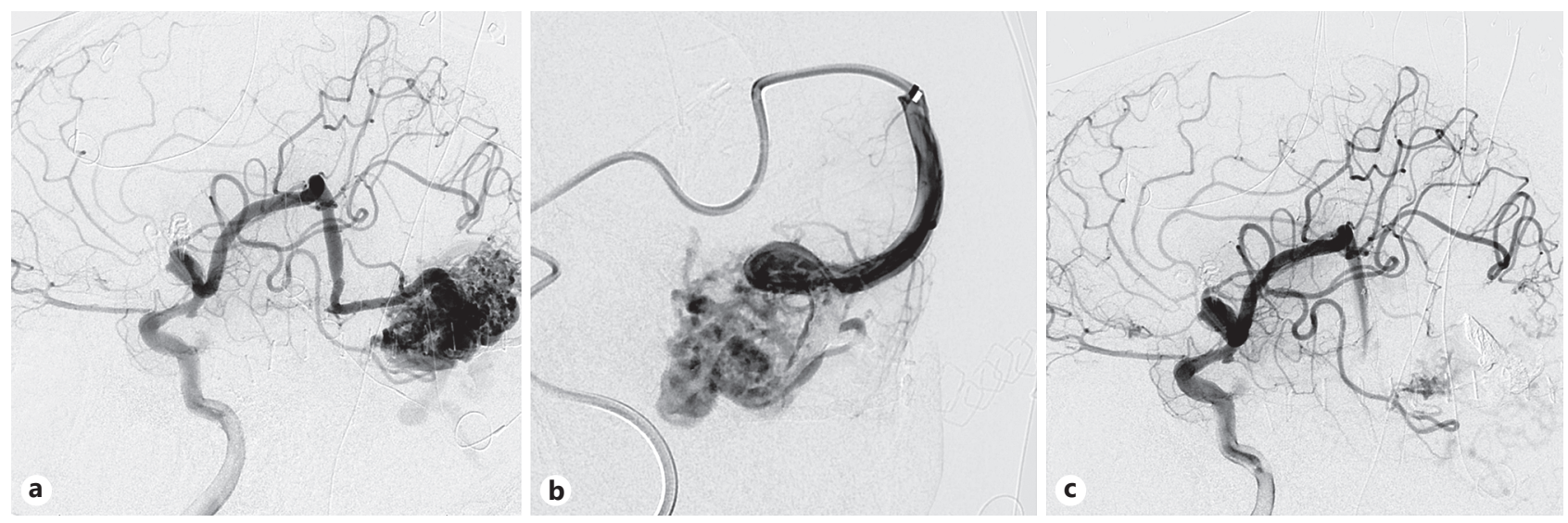

Fig. 2. This arteriovenous malformation (a, lateral angiographic view of internal carotid artery injection) was embolized using a 5F SOFIA catheter advanced to the M3 over the 2.1-Fr microcatheter utilized to carry out the embolization (b, superselective run via the SOFIA; c, control angiography after embolization). 


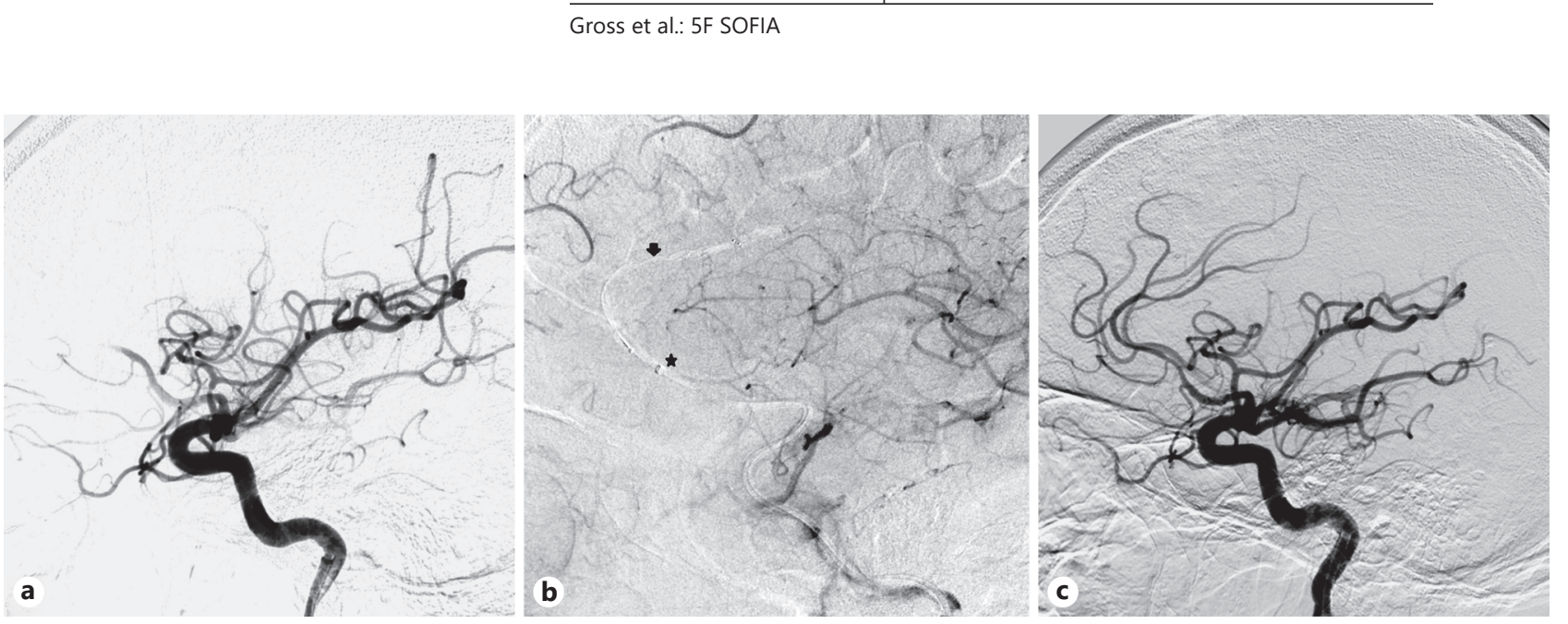

Fig. 3. This symptomatic distal A2 occlusion (a, lateral angiographic view of internal carotid artery injection) was treated via stent-assisted manual aspiration thrombectomy. A 4-mm stent (b, arrow) was deployed, over which the 5F SOFIA was advanced (b, star) into the anterior cerebral artery. An angiographic run after thrombectomy demonstrated interval recanalization (c, lateral angiographic view of internal carotid artery injection after thrombectomy).

\section{Arteriovenous Shunt Embolization}

The SOFIA was utilized in 4 arteriovenous fistula embolizations and 6 arteriovenous malformation embolizations. For the 4 fistulas, the SOFIA was advanced over a 2.1-Fr headway duo $(n=3)$ or a scepter balloon $(n=1)$ utilized for the embolization. Target locations were V3/4 $(n=3)$ and the distal occipital artery $(n=1)$. For the 6 arteriovenous malformations, the SOFIA was advanced over 2.1-Fr microcatheters (headway duo, $n=5$; echelon-10, $n=1$ ). Target locations were the distal basilar artery $(n=2), \mathrm{P} 1(n=1), \mathrm{M} 1(n=1)$, and M2/3 $(n=2$, Fig. 2 as illustrative case).

\section{Stroke Thrombectomy}

The 5F SOFIA was utilized as an aspiration catheter for distal stroke thrombectomy in 5 cases with modified treatment in cerebral infarction (mTICI) $2 \mathrm{~b} / 3$ results achieved in 4 cases and a mTICI 2a result achieved in 1 case. In 2 cases, it was advanced over a deployed 4-mm stent into the A2 for successful stent-assisted manual aspiration thrombectomy (Fig. 3). In 3 cases, it was utilized for M2 thrombectomy: in 2 cases, it was advanced over a stent into the $\mathrm{M} 2$, and in 1 case, it was advanced over an 0.027 microcatheter for manual aspiration thrombectomy.

\section{Discussion}

The 5F SOFIA is a flexible 0.055-inch inner diameter support catheter with multiple applications relevant in both embolization and thrombectomy. Design features specifically focus on its soft distal shaft, allowing for easier navigation in tortuous vessels and enhanced "kink resistance" to allow for easier 1:1 push/pull control. The tip can be steam-shaped, and it is available in 115 or $125 \mathrm{~cm}$ lengths. Its relatively small outer diameter (5 Fr) is a salient design feature that makes it compatible with 6-Fr guide catheters with a maintained, relatively robust, 0.055 -inch inner diameter. Illustrative cases in this series (Fig. 1-3) highlight its broad applicability; interestingly, each was performed by a different primary operator. 


\section{Aneurysm and Arteriovenous Shunt Embolization}

The 5 F SOFIA harbors particular relevance in the embolization of distal aneurysms such as mycotic aneurysms and arteriovenous shunts requiring only one microcatheter. It can be advanced into distal vasculature such as the PCA or MCA (Fig. 2), while its large inner diameter allows for excellent quality control angiographic runs during the embolization. Reasonable alternatives for intermediate catheter support in the PCA or ACA with comparable catheter flexibility harbor substantially smaller catheter inner diameters $(0.038$ inch or 0.044 inch). For pipeline embolization of more distal aneurysms, the SOFIA is easily advanced over 0.027inch microcatheters to either the ACA or MCA for added support. Its compatibility with a 6-Fr guide catheter can bear particular relevance in cases with challenging access anatomy requiring triaxial (Fig. 1) or even, if needed, quadraxial systems with 8-Fr sheaths. For complex proximal aneurysms, the SOFIA is well-suited for advancement over the delivery microcatheter past the aneurysm for extremely stable flow diverter deployment.

\section{Stroke Thrombectomy}

In the spirit of continuing to "push the limits" of applicability of stroke thrombectomy, it has become relatively commonplace to treat symptomatic M2 or even M3 occlusions [3, 4]. Recent literature has reported the usage of an 0.035-inch aspiration catheter to treat these occlusions [5, 6]. In our experience, proximal M2 occlusions that are still along the sphenoidal MCA, at times anatomically referred to as the "post bifurcation M1," are often treated with 0.060 -inch catheters. True insular M2 occlusions beyond the limen insulae often mandate smaller aspiration catheters; we have historically utilized 0.041 -inch or 0.044 -inch catheters for this purpose. The SOFIA catheter strikes a nice balance between its smaller outer diameter ( $5 \mathrm{Fr}$ ) and relatively large inner diameter ( $0.055 \mathrm{inch}$ ) that is well-suited for M2 occlusions. Other distal access catheters with comparable inner diameters have considerably larger outer diameters (at least 5.4 Fr) and, in our hands, are subjectively considerably less supple than the SOFIA. We also demonstrate the feasibility of bringing the SOFIA into the ACA over both a 0.027 -inch microcatheter (Fig. 1b) or a stent (Fig. 3b), particularly relevant considerations for ACA thrombectomy.

\section{Study Limitations}

Limitations of this study include its small retrospective design along with obvious selection bias for the usage of SOFIA in well-selected cases. We have just begun to explore the utility of the 5F SOFIA in stroke thrombectomy and, thus, cannot make conclusive remarks about its utility in M2 occlusions and particularly its trackability to the clot based on the small number of cases in this cohort. While the SOFIA is very soft and relatively easily brought to its desired target, its softer design can be a limitation in selected flow diversion cases, where a stiffer and/or larger support catheter is desired.

\section{Conclusion}

The unique dimensions of the relatively supple 5F SOFIA make it ideal as an adjunct for aneurysm and arteriovenous shunt embolization along with distal thrombectomy. 


\section{Disclosure Statement}

B.T.J. is a consultant for Medtronic. T.G.J. is a consultant for Neuravi, Codman Neurovascular, Stryker (PI DAWN; unpaid), and Fundacio Ictus (PI REVASCAT; unpaid), and holds stock in Anaconda, Silk Road, and Blockade. This research received no specific grant from any funding agency in the public, commercial, or notfor-profit sectors.

\section{Author Contributions}

Drafting the article: B.A.G. Acquisition of data / data analysis: B.A.G., W.J.A., and C.L.K. Reviewed and revised article prior to submission: all authors. Study supervision: B.A.G. and B.T.J.

\section{References}

1 Mohlenbruch MA, Kabbasch C, Kowoll A, et al: Multicenter experience with the new SOFIA plus catheter as a primary local aspiration catheter for acute stroke thrombectomy. J Neurointerv Surg 2016; DOI 10.1136/ neurintsurg-2016-012812.

-2 Heit JJ, Wong JHY, Mofaff AM, et al: Sofia intermediate catheter and the SNAKE technique: safety and efficacy of the Sofia catheter without guidewire or microcatheter support. J Neurointerv Surg 2017; DOI 10.1136/ neurintsurg-2017-013256.

-3 Jankowitz BT, Grandhi R, Horev A, et al: Primary manual aspiration thrombectomy (MAT) for acute ischemic stroke: safety, feasibility and outcomes in 112 consecutive patients. J Neurointerv Surg 2015;7:27-31.

4 Saber H, Narayanan S, Palla M, et al: Mechanical thrombectomy for acute ischemic stroke with occlusion of the M2 segment of the middle cerebral artery: a meta-analysis. J Neurointerv Surg 2017; DOI 10.1136/ neurintsurg-2017-013515.

5 Navia P, Larrea JA, Pardo E, et al: Initial experience using the 3MAX cerebral reperfusion catheter in the endovascular treatment of acute ischemic stroke of distal arteries. J Neurointerv Surg 2016;8:787-790.

6 Altenbernd J, Kuhnt O, Hennigs S, et al: Frontline ADAPT therapy to treat patients with symptomatic M2 and M3 occlusions in acute ischemic stroke: initial experience with the Penumbra ACE and 3MAX reperfusion system. J Neurointerv Surg 2017; DOI 10.1136/neurintsurg-2017-013233. 